\title{
Functionalization of PE nonwoven Fabric by Plasma Treatment to improve Dyeing Affinity
}

\author{
Ichiro Enomoto $^{1}$, Kazuya Mishima ${ }^{2}$, Takashi Kobayashi ${ }^{2}$ and Shin Soeda ${ }^{1}$ \\ ${ }^{I}$ Tokyo Metropolitan Industrial Technology Research Institute, \\ KFC bldg., 12F, 1-6-1 Yokoami, Sumida-ku, Tokyo 130-0015 Japan \\ ${ }^{2}$ NIPPO ELECTRONICS CO., LTD, \\ 8-1 Minamikurokawa Asao-ku Kawasaki-city Kanagawa 215-0034 Japan
}

\begin{abstract}
When the surface of ultra-high molecular weight polyethylene (UHMWPE) nonwoven fabric was treated with an oxygen plasma, the hydrophilic nature of the sample surface was improved. However, the sample was only colored slightly by in a dyeing test with dyestuffs done to investigate the effect of surface functionalization. A mixed-gas plasma treatment with fluorocarbon was able produce a sample that could be dyed a dark color using acid and reactive dyes. From the results of fourier transform infrared spectroscopy (FT-IR) and X-ray photoelectron spectroscopy (XPS) measurements, it was found that the carboxyl group increased on the sample surface as a result of the mixed-gas plasma treatment. As a simple evaluation method for investigating the effect of plasma treatment, the dyeing test was effective.
\end{abstract}

Keywords: plasma treatment, FT-IR, XPS, dyeing test

\section{Introduction}

Ultra-high molecular weight polyethylene (UHMWPE) has a high strength-to-weight ratio and, has excellent chemical resistance and wears resistance. As a result of these characteristics, UHMWPE is used in industrial and medical applications [1]. Nonwoven UHMWPE fabric is used for electrolyte films, such as is found in lithium ion batteries; UHMWPE fiber is used for fishing lines and towing ropes; and the molded form of UHMWPE is used for orthopedic artificial joint material. However, because of the lack of a reactive group on the polyethylene backbone or low surface energy, the surface is hydrophobic and lacks adhesive properties and dyeing affinity. For these reasons, surface treatments have been applied to modify this polymer.

To improve the wettability and adhesion of hydrophobic polymer material such as UHMWPE by plasma and UV treatments [2-5], $\gamma$-ray irradiation [6-9] and electron beam irradiation [10] have been used.
In particular, surface treatment with plasma yields improved results. It is also an excellent technology from an environmental and energy-saving perspective because minimal hazardous wastes are discharged and compared with general wet processing, processing and waste fluid is unnecessary. The depth of the layer that is changed by plasma treatment is hundreds of micrometers from the surface; the character of the bulk material is not changed. In addition, it is possible to improve the wettability in a short time, and it is very effective as a printing pretreatment for the purpose of improving adhesion. In general, the water drop contact angle test is performed to measure the effect of the surface treatment.

It is known that the hydrophilic layer (polar group) generated on the surface will decrease with time. Graft polymerization is an excellent method for maintaining the hydrophilic effect for a prolonged period. However, workability is a problem, along with contamination in the equipment. \begin{tabular}{lll}
\hline Received & May & 14,2010 \\
Accepted & June & 11,2010
\end{tabular} 
In this research study, the plasma treatment conditions were examined to maintain the hydrophilic layer and the functionality on the polymer surface. A dyeing evaluation was performed as a simple method of checking the effect.

\section{Experimental}

\subsection{Materials and plasma treatment}

A UHMWPE nonwoven sample with a thickness of $50 \mu \mathrm{m}$ was used (Teijin Co., Ltd. 7P03A).

A plasma treatment apparatus (PCB-2800 made by March) was used with various gases. It used an electric discharge output power of 2000 to $5000 \mathrm{~W}$, a pressure of $53 \mathrm{~Pa}$, and a 15 to 60 minutes processing times.

2.2. Fourier transform infrared spectroscopy (FT-IR) and X-ray photoelectron spectroscopy (XPS) measurements

To investigate the chemical constitution change of the samples after the plasma treatment, an FTIR 4300 by Jasco Corp. was used. The scans were performed with the transmission method using eight laminated nonwoven fabrics. To investigate the surface chemical constitution change in detail, the attenuated total reflectance (ATR) method was also utilized.

In a similar way, an ESCA 5600Ci (Mg-K alpha rays, $1253.6 \mathrm{eV}$ ) made by Ulvac Corp. was used for XPS analysis. It had an incorporation angle of 45 degrees, a beam diameter of $800 \mu \mathrm{m}$, a vacuum level of $1.3 \times 10^{-7}$ to $1.3 \times 10^{-6} \mathrm{~Pa}$, and an analysis time of $5 \mathrm{~min}$.

\subsection{Dyeing test}

The plasma treated samples were dyed using a 0.01 to $0.16 \%$ solution of acid dye (Kayanol Milling Red BW) and reactive dye (Remazol Red RU-N). Dyeing of the samples with the acid dye was done at $90^{\circ} \mathrm{C}$ for $30 \mathrm{~min}$ in a pH 6 to 7 . Dyeing of the samples with the reactive dye was done at $60^{\circ} \mathrm{C}$ for $30 \mathrm{~min}$. The reactive dye solution was prepared with $50 \mathrm{~g} / \mathrm{L}$ of anhydrous sodium sulfate acetic acid and $20 \mathrm{~g} / \mathrm{L}$ of anhydrous sodium carbonate.

In a simple test, the sample was dipped in an approximately $0.02 \%$ dye solution for 5 minutes, and the grade of coloring was investigated visually.

2. 4 Scanning electron microscopy (SEM) and tensile strength test
The surface structures of untreated and plasma treated samples were observed with a JSM-5600LV (JEOL Ltd.) at $3.0 \mathrm{keV}$. The samples were coated with a thin layer of gold for scanning electron microscopy observation.

To investigate the change of tensile strength after treatment, a tensile tester (RTM500) made by Orientech Corp. was used with a sample length of $80 \mathrm{~mm}$, a tensile speed of $50 \mathrm{~mm} / \mathrm{min}$, and a load cell of $50 \mathrm{~N}$.

\section{Results and discussion}

The chemical constitution of PE nonwoven fabric changed with the oxygen plasma treatment. The results of the FT-IR measurements are shown in Fig. 1. After oxygen plasma treatment, the peak of the oxide was seen around $1750 \mathrm{~cm}^{-1}$.

a) untreated

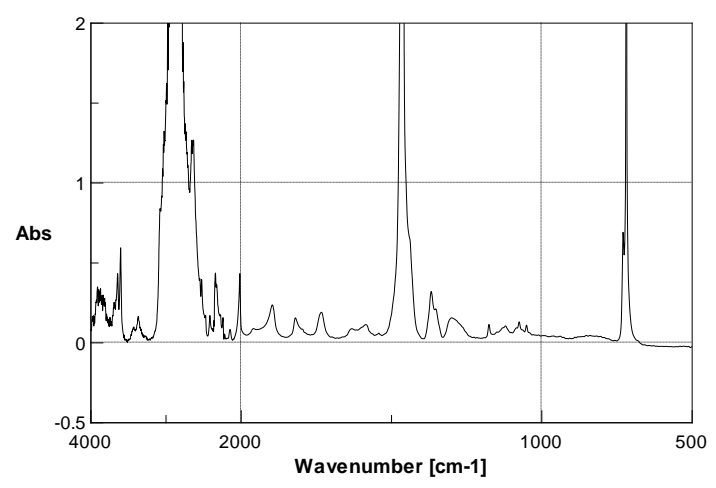

b) oxygen plasma treatment

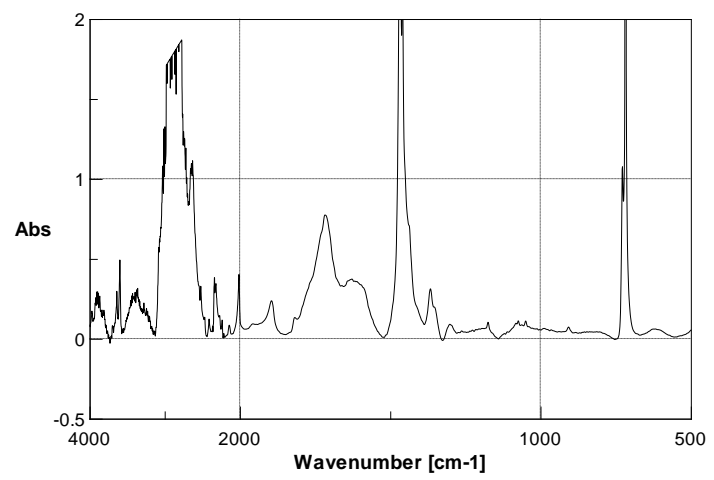

Figure 1. FT-IR spectra of UHMWPE nonwoven fabric a) untreated, b) oxygen plasma treatment $(2500 \mathrm{~W}, 53 \mathrm{~Pa}, 120 \mathrm{sec})$. The peak of the oxide was seen to around $1750 \mathrm{~cm}^{-1}$.

Because the effect of surface treatment using direct fluorocarbons [11-12] and fluorocarbon plasma [13-15] have been reported, an oxygen plasma treatment with $\mathrm{CF}_{4}$ gas was considered. The results of the FT-IR measurement $\mathrm{s}$ are shown in Fig. 2. The peaks of (1) and (3) are 
considered to be $-\mathrm{OH}$, and the peak of (2) is considered to be $-\mathrm{COOH}$ or $-\mathrm{C}=\mathrm{C}-$. Because it was not possible to specify a product using only the results of the FT-IR measurements, XPS measurements were performed. The results are shown in Fig. 3. The peak of (1) and (2) were determined to be $-\mathrm{COOH}$ and $-\mathrm{C}-\mathrm{OH}$, respectively.

a) untreated

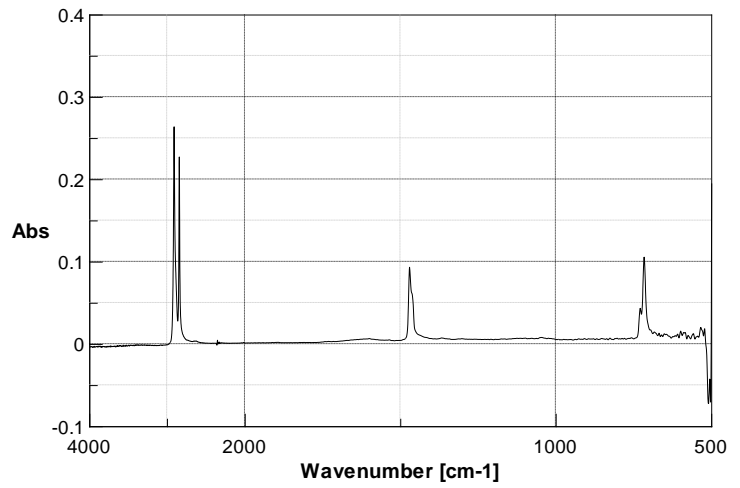

b) mixed-gas plasma treatment

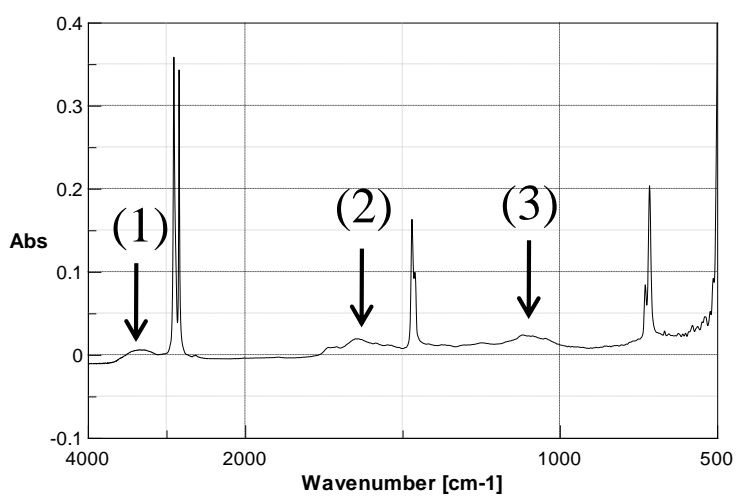

Figure 2. FT-IR (ATR) spectra of UHMWPE nonwoven fabric a) untreated, b) mixed gas plasma treatment $\left(\mathrm{O}_{2} 90 \%, \mathrm{CF}_{4} 10 \%, 2000 \mathrm{~W}, 53 \mathrm{~Pa}, 15\right.$ sec). The peak of (1) and (3) are considered to be $-\mathrm{OH}$, and the peak of (2) is considered to be $-\mathrm{COOH}$ or $-\mathrm{C}=\mathrm{C}-$.

The SEM images of oxygen gas plasma treatment and those of the mixed-gas plasma treatment with $\mathrm{CF}_{4}$ gas are shown in Fig. 4 . In the oxygen gas plasma treatment, signs that the crevices between nonwoven fibers were lost by anastomosis were observed. If the fact that the melting temperature of a sample is around $130^{\circ} \mathrm{C}$ is taken into consideration, it appears that this temperature was exceeded during the plasma treatment. a) untreated

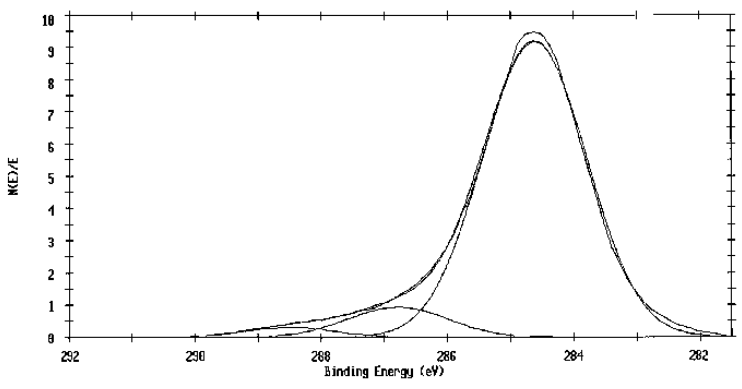

b) mixed-gas plasma treatment

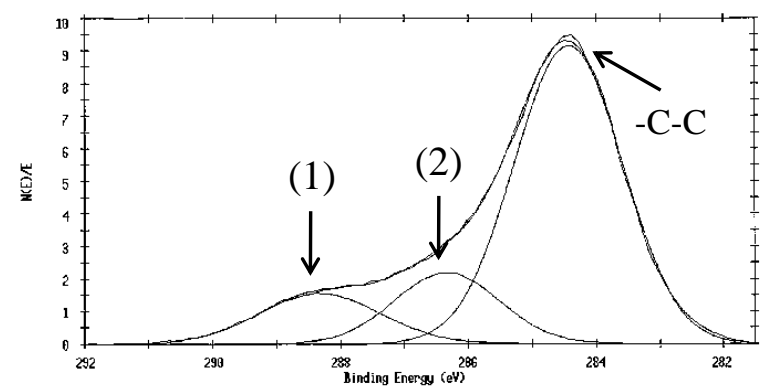

Figure 3. XPS spectra of UHMWPE nonwoven fabric a) untreated, b) mixed gas plasma treatment $\left(\mathrm{O}_{2} 90 \%, \mathrm{CF}_{4} 10 \%, 2000 \mathrm{~W}, 53 \mathrm{~Pa}, 15 \mathrm{sec}\right)$. The peak of (1) and (2) considered to be $-\mathrm{COOH}$ and $-\mathrm{C}-\mathrm{OH}$, respectively.

Although a state like anastomosis was not seen in the mixed-gas plasma treatment with $\mathrm{CF}_{4}$ (15s), when the processing time became long (30 s), super-thin fibers were removed by etching, and the crevices increased. Furthermore, when the processing time was lengthened (60 s), contractions increased considerably.

The sample dyed after oxygen plasma treatment achieved the grade "slightly colored" from the acid dye and the reactive dye. However, after processing with the mixed gas including $\mathrm{CF}_{4}$ both dyes were able to dye the treated sample.

It is thought that the tensile strength of a PE sample is decreased by plasma treatment. For this reason, the tensile strength of the sample before and after plasma treatment was investigated. It decreased to half of the initial strength after oxygen plasma treatment and to about $60 \%$ of the initial strength after the mixed-gas plasma treatment.

Although each processing condition chosen for this research study performs well in the water drop contact angle test, other methods were needed to evaluate the specific functionalization. The effect of the surface treatment was inves- 
a)

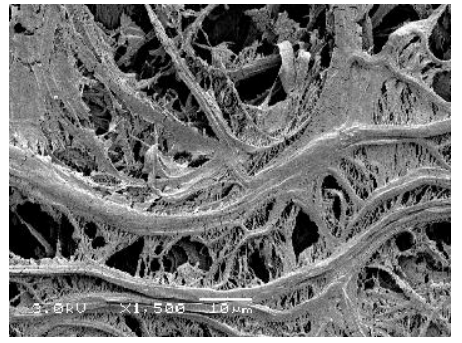

b)

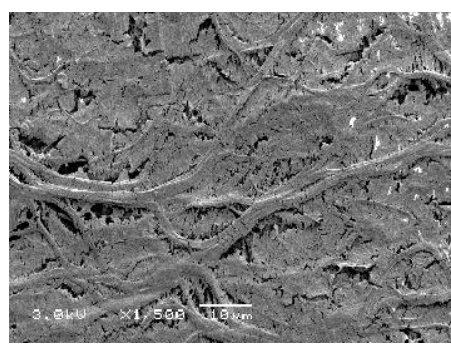

c)

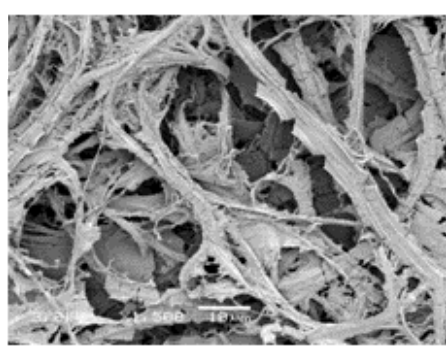

Figure 4. SEM images of UHMWPE nonwoven fabric a) untreated, b) oxygen plasma treated (2500 $\mathrm{W}, 53 \mathrm{~Pa}, 120 \mathrm{sec})$, c) mixed gas plasma treatment $\left(\mathrm{O}_{2} 90 \%, \mathrm{CF}_{4} 10 \%, 2000 \mathrm{~W}, 53 \mathrm{~Pa}, 15 \mathrm{sec}\right)$.

tigated using FT-IR, XPS, and SEM. Although effects such as generation of a hydrophilic and an oxide were seen after the plasma treatment under an oxygen gas atmosphere, the dyeing affinity was not high enough because of a lack of functionality on the sample surface. Because the surface was melting during this treatment, the processing conditions could not be made any more severe. The sample treated with the mixed-gas plasma treatment with fluorocarbon was able to be considerably dyed with the dark color using the acid and reactive dyes. However, if the dye concentration became high, an untreated sample was also dyed a dark color.
Concerning the mechanism of dyeing, more experiments are clearly required.

\section{Conclusions}

The dyeing test with dyestuffs was effective as an advanced method to compare with the water drop contact angle test for investigating surface functionalization using a plasma treatment. However, the dyeing mechanism is still not fully understood.

\section{Reference}

1. S. M. Kurtz, O. K. Muratoglu, M. Evans and A. A. Edidin, Biomaterials. 20 (1999). 1659.

2. S. I. Moon and J. Jang, J. Appl. Polym. Sci., 68 (1998), 1117.

3. S. I. Moon and J. Jang, J. Adhesion Sci. Technol., 14 (2000), 493.

4. M. Mori, Y. Uyama and Y. Ikada, J. Polym. Sci. Part A: Polym. Chem., 32 (1994), 1683.

5. J. M. Yang, P.Y. Huang, M. C. Yang and W. Wang, J. Appl. Polym. Sci., 65 (1997) 365.

6. K. Kaji, Y. Abe, M. Murai, N. Nishioka and K. Kosai, J. Appl. Polym. Sci., 47 (1993), 1427.

7. A. B. Brennan, J. J. Arnold and M. P. Zamopa, J.Adhesion Sci. Technol., 9 (1995), 1031.

8. O. H. Kwon, Y. C. Nho, J. H. Jin, M. J. Lee and Y. M. Lee, J. Appl. Polym. Sci., 72 (1999), 659.

9. I. Enomoto, Y. Katsumura, H. Kudo and M. Sekiguchi, Radiat. Phys. Chem., 79 (2010), 718.

10. K. Sakurai, Y. Kondo, K. Miyazaki, Y. Okamoto, S. Irie and T. Sasaki, J. Polym. Sci. Part B: Polym. Phys., 42 (2004), 2595.

11. A. J. Otsuka and R. J. Lagow, J. Fluor. Chem., 4 (1974), 371.

12. A. P. Kharitonov and Y. L. Moskvin, J. Fluor. Chem., 91 (1998), 87.

13. M. Strobel, S. Corn, C. S. Lyons and G. A Korba, J. Polym. Sci., 23 (1985), 1125.

14. M. Strobel, S. Corn, C. S. Lyons and G. A Korba, J. Polym. Sci., Part A. 25 (1987), 1295.

15. Y. Iriyama and H. Yasuda, J. Polym. Sci., Part A. 30 (1992), 1731. 